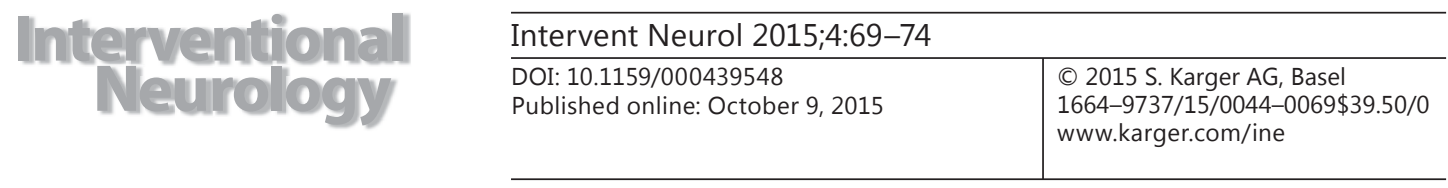

\title{
Hemorrhagic Transformation in Patients with Cerebral Infarction Referred to a Rehabilitation Hospital
}

\author{
Shinichiro Maeshima Sayaka Okamoto Hideto Okazaki Shiho Mizuno \\ Naoki Asano Tetsuya Tsunoda Hirofumi Maeda Mitsuko Masaki \\ Shigeru Sonoda \\ Department of Rehabilitation Medicine II, School of Medicine, Fujita Health University, Tsu, Japan
}

\section{Key Words}

Cerebral infarction $\cdot$ Complication $\cdot$ Hemorrhage

\begin{abstract}
Purpose: The aim of our study was to investigate the presenting conditions of patients with hemorrhagic transformation (HT), the frequency of $\mathrm{HT}$ and its treatments at a rehabilitation hospital. Subjects and Methods: 165 patients with cerebral infarctions transferred to our rehabilitation hospital during the study period were enrolled. HT was diagnosed by two stroke specialists using computed tomography (CT) at the time of transfer to our rehabilitation hospital and other imaging data from previous consultations. Neurological status, activities of daily living, administered agents, patient age, duration from stroke onset to transfer and length of hospital stay were examined. Results: 30 of the 165 patients (18.2\%) were diagnosed with HT. Decreased activities of daily living at admission and discharge as well as longer hospitalization stays were more prevalent in HT patients than in those without HT. We did not change antiplatelet or anticoagulant agents, but continued rehabilitation over a defined period while monitoring blood pressure; we confirmed with $\mathrm{CT}$ that the patients' physical conditions did not deteriorate. Conclusion: With careful symptom and CT monitoring over a defined period, rehabilitation can be continued in patients who develop HT at the time of transfer to a rehabilitation hospital in the acute phase of cerebral infarction.
\end{abstract}

(C) 2015 S. Karger AG, Basel 


\section{Introduction}

Hemorrhagic transformation (HT), which refers to a spectrum of ischemia-related brain hemorrhages, is a complication of ischemic stroke. It occurs in about $10 \%$ of patients with cerebral infarction $[1,2]$. There are two types of HT: one occurs within a few days following an acute cerebral infarction, and the other occurs during the sub-acute phase (approximately 1 week to 1 month following the event) [3, 4]. Many HTs occur in an acute care hospital. A patient with HT already displays some neurological symptoms due to the ischemic stroke, and the medical staff may not notice the change in the patient's status. The patient is often transferred to a rehabilitation hospital without the HT being diagnosed. The aim of our study was to investigate the presenting conditions of patients with HT at a rehabilitation hospital, the rate of HT and the treatment methods.

\section{Subjects and Methods}

232 patients with cerebral infarctions were transferred to the recovery rehabilitation ward at our hospital between April 1, 2013 and March 31, 2014. Of these, 165 were enrolled in this study, excluding 47 patients with recurrent cerebral infarction, 12 patients who had undergone surgery and 8 patients with unavailable medical information (fig. 1). The age of the patients (102 men, 63 women) ranged from 36 to 93 years. The duration from stroke onset to transfer to a recovery rehabilitation hospital was 10-64 days. The average length of hospital stay was $59.2 \pm 34.1$ days (range 10-210 days).

The disease types based on Trial of Org 10172 in acute stroke treatment [5] were as follows: small vessel occlusion (13 patients, Essen Stroke Risk Score [ESRS] $3.0 \pm 1.5$ ), large artery atherosclerosis (76 patients, ESRS $3.3 \pm 1.2$ ), cardioembolism (48 patients, $\mathrm{CHADS}_{2}$ score $3.4 \pm 0.8$ ) and other types (28 patients). Based on the Oxfordshire Community Stroke Project [6], the patients' pathological changes were classified into the following groups: total anterior circulation infarct $(n=15)$, partial anterior circulation infarct $(n=77)$, lacunar infarct $(n=28)$ and posterior circulation infarct $(n=45) .91$ patients had previously been treated with one antiplatelet agent, 17 patients with two types of antiplatelet agents, 47 patients with an anticoagulant agent and 3 patients with both an anticoagulant agent and an antiplatelet agent; 7 patients had no history of treatment with these drugs.

The following factors were examined for each patient: Canadian Neurological Scale (CNS) score [7] at first visit, Mini-Mental State Examination (MMSE) score [8], activities of daily living at admission and discharge using the Functional Independence Measure (FIM) [9], use of thrombolytic therapy with tissue plasminogen activator (tPA) and prescription drugs, patient age, time from onset of stroke to transfer and length of hospital stay. HT was diagnosed by two stroke specialists using computed tomography (CT) findings at the time of transfer to our rehabilitation hospital and other imaging data from previous consultations. HT

Fig. 1. Flow chart of patient re-

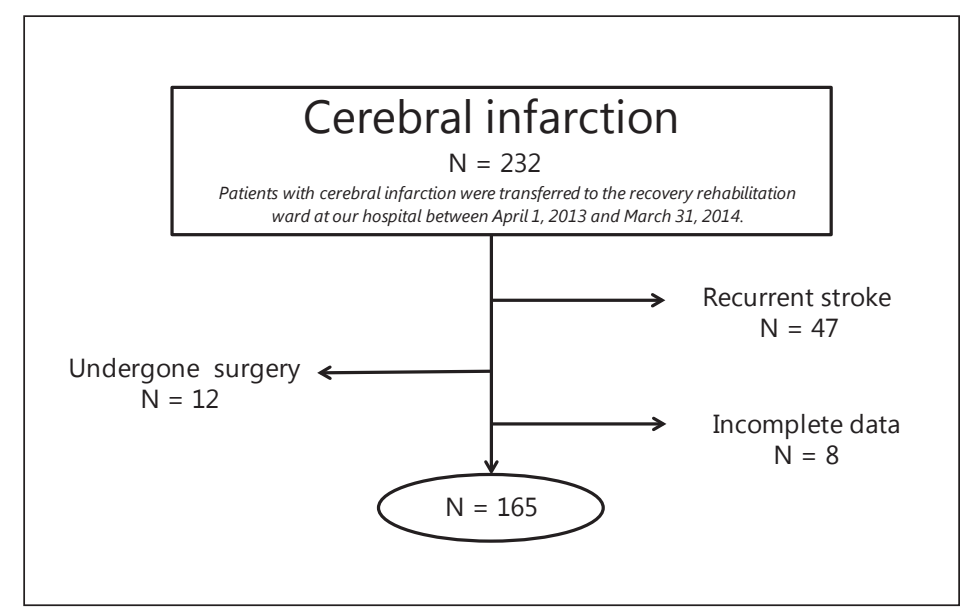
cruitment. 
was classified according to the European Cooperative Acute Stroke Study (ECASS) guidelines [10]. The patients with HT were then further classified into two groups: (1) patients who developed HT within 1 week following the onset of cerebral infarction (early group) and (2) patients who developed HT more than 1 week after onset (delayed group). These two groups were compared with the group without HT.

Statistical analyses were performed with JMP 9.02. The $\chi^{2}$ test for independence was used to determine whether there was a significant association between two variables. The Kruskal-Wallis test was used to compare three groups of sample data and to determine any association among them. When a statistical significance of 5\% was achieved, a multiple comparison based on the Steel-Dwass test was performed.

This study was approved by the institutional review board of our university. Detailed explanations were given to the participants and informed consent was obtained from all patients.

\section{Results}

30 of the 165 patients (18.2\%) were diagnosed with HT: 12 patients were classified as belonging to the early group, while 18 were classified as belonging to the delayed group (fig. 2). There was no association between the time of HT development and the ECASS classification. We observed no deterioration in patients' symptoms.

No statistical differences were found among the three groups (the early group, the delayed group and the group without HT) in age, sex, lesion side, CNS score or duration from onset to hospitalization (table 1). However, there were significant differences in the type of cerebral infarction, lesion classification and tPA treatment. HT was not detected in any patient with lacunar infarction; however, $15.8 \%$ of patients with atherothrombotic cerebral infarctions and $31.3 \%$ of those with cerebral embolisms were diagnosed with HT. There were also differences in MMSE score, number of hospitalization days, FIM at admission and discharge, FIM efficiency and time until discharge. In particular, reduction of FIM at admission and discharge and longer hospitalization were noted in HT patients in the early phase than in those with HT in the delayed phase or without HT. 12 patients had been prescribed tPA previously, and 6 of these patients were diagnosed with HT. There were no differences in age, sex, lesion location, type of cerebral infarction, CNS score, duration from cerebral infarction onset to hospitalization, neurological symptoms and activities of daily living at admission and discharge between tPA-treated patients and non-tPA-treated

Fig. 2. Duration from stroke onset to development of HT. Horizontal lines show median number of days from stroke onset to development of HT.

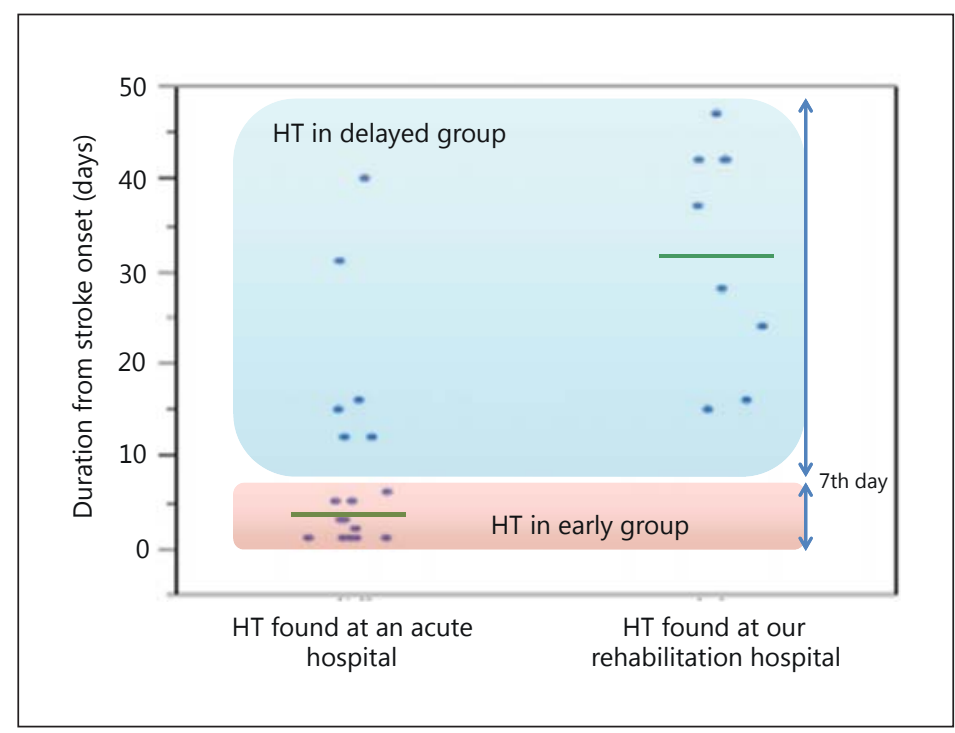


Maeshima et al.: Hemorrhagic Transformation in Patients with Cerebral Infarction

Referred to a Rehabilitation Hospital

Table 1. Demographic, clinical and morphological variables for early or delayed HT versus no HT

\begin{tabular}{|c|c|c|c|c|}
\hline & Early HT & Delayed HT & No HT & $\mathrm{p}$ value \\
\hline Duration from stroke onset to HT, days & $1(1$ to 6$)$ & 21 (5 to 47$)$ & - & $<0.0001^{\mathrm{a}}$ \\
\hline ECASS classification HI-1/HI-2/PH-1/PH-2 & $3 / 4 / 0 / 5$ & $4 / 6 / 2 / 6$ & - & $0.5316^{\mathrm{b}}$ \\
\hline Age, years & 71 (37 to 89$)$ & $72(36$ to 86$)$ & 70 (37 to 93$)$ & $0.7300^{\mathrm{a}}$ \\
\hline Sex, male/female & $8 / 4$ & $15 / 3$ & $79 / 56$ & $0.0891^{\mathrm{b}}$ \\
\hline Oxford, LI/PACI/PCI/TACI & $0 / 9 / 0 / 3$ & $0 / 10 / 6 / 2$ & $28 / 58 / 39 / 10$ & $0.0007^{\mathrm{b}}$ \\
\hline TOAST, LAA/SVO/CE/others & $6 / 0 / 4 / 2$ & $6 / 0 / 11 / 1$ & $64 / 13 / 33 / 25$ & $0.0296^{\mathrm{b}}$ \\
\hline Lesion side, right/left/bilateral & $5 / 7 / 0$ & $8 / 10 / 0$ & $58 / 73 / 4$ & $0.7982^{b}$ \\
\hline With tPA/without tPA & $3 / 9$ & $3 / 15$ & $6 / 129$ & $0.0273^{\mathrm{b}}$ \\
\hline CNS, /11.5 & 5 (3 to 11$)$ & 7 (3 to 10.5$)$ & 8 (3 to 11.5$)$ & $0.0786^{\mathrm{a}}$ \\
\hline MMSE, /30 & 23.5 (1 to 28$)$ & $22(9$ to 28$)$ & $26(5$ to 30$)$ & $0.0302^{\mathrm{a}}$ \\
\hline Duration from stroke onset to rehabilitation, days & $28(17$ to 54$)$ & 36.5 (15 to 50$)$ & $28(10$ to 64$)$ & $0.1717^{\mathrm{a}}$ \\
\hline Length of stay at rehabilitation hospital, days & $84(15 \text { to } 150)^{*}$ & 56.5 (22 to 189$)$ & 48.5 (10 to 210$)$ & $0.0224^{\mathrm{a}}$ \\
\hline FIM score on admission, /126 & 46.5 (18 to 82$)^{*}$ & $45(18$ to 110$)$ & $73(19$ to 126$)$ & $0.0055^{\mathrm{a}}$ \\
\hline FIM score at discharge, /126 & $68(24 \text { to } 100)^{*}$ & $67(21 \text { to } 116)^{*}$ & 104 (19 to 126$)$ & $0.0014^{\mathrm{a}}$ \\
\hline FIM gain & $16.5(-25$ to 47$)$ & $13.5(0$ to 49$)$ & $19(-13$ to 86$)$ & $0.2037^{\mathrm{a}}$ \\
\hline FIM efficiency & $0.22(-0.26 \text { to } 0.44)^{*}$ & $0.17(0$ to 1.09$)$ & $0.37(-0.8$ to 2.1$)$ & $0.0084^{\mathrm{a}}$ \\
\hline Destination, home/others & $6 / 6$ & $12 / 6$ & $112 / 23$ & $0.0210^{\mathrm{b}}$ \\
\hline
\end{tabular}

$\mathrm{CE}=$ Cardioembolism; LAA = large artery atherosclerosis; LI = lacunar infarct; Oxford = Oxfordshire Community Stroke Project; $\mathrm{PACI}=$ partial anterior circulation infarct; $\mathrm{PCI}=$ posterior circulation infarct; $\mathrm{SVO}=$ small vessel occlusion; TACI $=$ total anterior circulation infarct; TOAST $=$ Trial of Org 10172 in acute stroke treatment. ${ }^{a}$ Kruskal-Wallis test. ${ }^{b} \chi^{2}$ test. ${ }^{*}$ p $<0.05$ versus HT.

Table 2. Differences in demographic and clinical variables between patients with and without tPA

\begin{tabular}{llll}
\hline & With tPA & Without tPA & p value \\
\hline Duration from stroke onset to HT, days & $5.5(1$ to 40$)$ & $13.5(1$ to 47$)$ & $0.4664^{\mathrm{a}}$ \\
ECASS classification HI-1/HI-2/PH-1/PH-2 & $1 / 1 / 2 / 2$ & $6 / 8 / 1 / 9$ & $70(36$ to 93$)$ \\
Age, years & $78(51$ to 90$)$ & $94 / 59$ & $0.2894^{\mathrm{b}}$ \\
Sex, male/female & $8 / 4$ & $27 / 69 / 44 / 13$ & $0.7173^{\mathrm{b}}$ \\
Oxford, LI/PACI/PCI/TACI & $1 / 9 / 1 / 2$ & $71 / 12 / 42 / 28$ & $0.1999^{\mathrm{b}}$ \\
TOAST, LAA/SVO/CE/others & $5 / 1 / 6 / 0$ & $64 / 85 / 4$ & $0.1159^{\mathrm{b}}$ \\
Lesion side, right/left/bilateral & $7 / 5 / 0$ & $8(3$ to 11.5$)$ & $0.4341^{\mathrm{b}}$ \\
CNS, /11.5 & $7.5(3$ to 11$)$ & $26(1$ to 30$)$ & $0.3948^{\mathrm{a}}$ \\
MMSE, /30 & $22(9$ to 29$)$ & $28(10$ to 64$)$ & $0.2781^{\mathrm{a}}$ \\
Duration from stroke onset to rehabilitation, days & $37(15$ to 58$)$ & $68(18$ to 210$)$ & $0.2429^{\mathrm{a}}$ \\
Length of stay at rehabilitation hospital, days & $62(22$ to 94$)$ & $99(19$ to 126$)$ & $0.6745^{\mathrm{a}}$ \\
FIM score on admission, /126 & $49(18$ to 111$)$ & $19(-13$ to 86$)$ & $0.0844^{\mathrm{a}}$ \\
FIM score at discharge, /126 & $76(21$ to 119$)$ & $0.34(-0.8$ to 2.1$)$ & $0.5347^{\mathrm{a}}$ \\
FIM gain & $13.5(-25$ to 47$)$ & $122 / 31$ & $0.3103^{\mathrm{b}}$ \\
FIM efficiency & $0.38(-0.27$ to 0.70$)$ & $8 / 4$ & $0.5216^{\mathrm{a}}$ \\
Destination, home/others & & & \\
\hline
\end{tabular}

$\mathrm{CE}=$ Cardioembolism; LAA = large artery atherosclerosis; LI = lacunar infarct; Oxford = Oxfordshire Community Stroke Project; PACI = partial anterior circulation infarct; PCI = posterior circulation infarct; $\mathrm{SVO}=$ small vessel occlusion; $\mathrm{TACI}=$ total anterior circulation infarct; TOAST $=$ Trial of Org 10172 in acute stroke treatment. ${ }^{a}$ Kruskal-Wallis test. ${ }^{b} \chi^{2}$ test.

patients (table 2). 9 patients were diagnosed with HT using CT images taken at the time of transfer to our rehabilitation hospital. Although we did not change the antiplatelet or anticoagulant agents, we continued rehabilitation with CT over a defined period while monitoring blood pressure; we confirmed that the patients' physical conditions did not deteriorate. 


\section{Discussion}

In this study, $18.2 \%$ of the participants were diagnosed with HT; we included patients admitted for the purpose of rehabilitation within 2 months from stroke onset, while unconscious patients (severe stage) and those without neurological symptoms (mild stage) were excluded. (The Japanese national insurance system covers convalescent rehabilitation. Patients are eligible for admission to the convalescent rehabilitation ward within 2 months after the onset of disabling diseases, including stroke, traumatic brain injury, and other neurological diseases, as well as orthopedic diseases such as hip fracture. The maximal onsetadmission interval is 2 months and the maximal length of stay covered by the insurance is 150 days for stroke, 180 days for stroke and other neurological diseases with severe disability and cognitive disorders, and 90 days for orthopedic diseases and disuse/myopathy syndrome.) HT was most commonly detected in patients with cerebral embolisms; many patients exhibiting large lesions developed HT, while none of the patients with lacunar infarctions developed HT. These results are in line with those of previous reports $[4,11,12]$. Both HT groups showed reduction in FIM at admission and discharge compared with patients without HT. One possible explanation for this could be that many patients in these groups have large lesions and develop HT due to cerebral embolisms.

HT development in the acute phase is due to blood flow recirculation, which occurs when the clot that blocked the arterial blood flow to the brain moves [13], and reperfusion of occluded peripheral vessels via leptomeningeal anastomoses. This symptom is often detected within 5 days after cerebral infarction onset. On the other hand, HT development in the sub-acute phase is mainly due to abnormal vascular permeability and an increase in blood flow, which follows diminished cerebral edema [4]. In these cases, petechiae in the affected cerebral tissues and splinter hemorrhages along the cerebral cortex are detected. In our study, the presence of petechiae was not associated with the time of onset. Massive hematomas were also detected in our study. Previous studies have shown that a higher number of massive hematomas and a significant increase in mortality were detected in patients receiving tPA treatment (ECASS) than those who did not receive this treatment. However, the development of HT without massive hematoma was not associated with worsening neurological symptoms and there was also no association between this type of HT and functional prognosis $[14,15]$. Our study showed no difference in neurological symptoms and functional prognosis between patients treated with and without tPA, although HT developed with a higher incidence in patients treated with tPA. HT causes hemorrhage in the ischemic cerebral tissues. Patients undergoing rehabilitation already display some neurological symptoms due to cerebral infarction. Therefore, mild hemorrhage in the affected cerebral tissues does not always correlate with clinical outcome [16]. Paciaroni et al. [17] investigated the factors associated with worse functional prognosis after cerebral infarction in a multicenter cooperative study. They concluded that HT itself was likely to be an independent factor associated with poorer mortality and functional prognosis, unless hematoma was detected. On the other hand, Bayramoğlu et al. [18] showed that there was no association between HT development and functional prognosis, although HT was detected in $32.2 \%$ of patients with cerebral infarction who were admitted to a rehabilitation hospital in the acute phase.

When HT development and hematoma are detected during treatment, doctors cease the administration of anticoagulant agents. There was little information regarding the development of HT in the patients who participated in our study. The risk of recurrence was considered to be high based on $\mathrm{CHADS}_{2}$ score and ESRS, therefore we continued administration with great care, confirming the absence of both symptom deterioration and hematoma enlargement through the use of CT scans. Although we supposed that HT would cause physical 
therapists to be reluctant to plan an active rehabilitation program, we found that HT was less likely to inhibit factors associated with rehabilitation in this study.

The limitations of our study are that the subjects were hemiplegic patients in the subacute phase of stroke rehabilitation and that patients with mild or severe neurological symptoms were excluded. Furthermore, the time at which a CT scan was performed while patients were in the acute hospital was not consistent, because the condition of a patient did not change and a CT scan could not be taken every day. However, we conclude that rehabilitation can be continued for patients who have already developed HT at the time of transfer to a rehabilitation hospital in the acute phase of cerebral infarction, but careful observation of the symptoms and CT scans over a defined period must be maintained.

\section{Disclosure Statement}

The authors have no conflicts of interest to disclose.

\section{References}

1 Terruso V, D’Amelio M, Di Benedetto N, Lupo I, Saia V, Famoso G, Mazzola MA, Aridon P, Sarno C, Ragonese P, Savettieri G: Frequency and determinants for hemorrhagic transformation of cerebral infarction. Neuroepidemiology 2009;33:261-265.

2 Kim JT, Heo SH, Park MS, Chang J, Choi KH, Cho KH: Use of antithrombotics after hemorrhagic transformation in acute ischemic stroke. PLoS One 2014;9:e89798.

3 Kawase T, Mizukami M, Tazawa T, Araki G, Yunoki K, Nagata K: CT findings and pathologenetic mechanisms of hemorrhagic infarction. Prog Comput Imaging 1980;2:283-290.

4 Hornig CR, Dorndorf W, Agnoli AL: Hemorrhagic cerebral infarction - a prospective study. Stroke 1986;17: 179-185.

5 Adams HP Jr, Bendixen BH, Kappelle LJ, Biller J, Love BB, Gordon DL, Marsh EE III: Classification of subtype of acute ischemic stroke. Definitions for use in a multicenter clinical trial. TOAST. Trial of Org 10172 in Acute Stroke Treatment. Stroke 1993;24:35-41.

6 Bamford J, Sandercock P, Dennis M, Burn J, Warlow C: Classification and natural history of clinically identifiable subtypes of cerebral infarction. Lancet 1991;337:1521-1526.

7 Côté R, Hachinski VC, Shurvell BL, Norris JW, Wolfson C: The Canadian Neurological Scale: a preliminary study in acute stroke. Stroke 1986;17:731-737.

8 Folstein MF, Folstein SE, McHugh PR: 'Mini-Mental State': a practical method for grading the cognitive state of patients for the clinician. J Psychiatr Res 1975;12:189-198.

9 Granger CV, Hamilton BB, Linacre JM, Heinemann AW, Wright BD: Performance profiles of the functional independence measure. Am J Phys Med Rehabil 1993;72:84-89.

10 Fiorelli M, Bastianello S, von Kummer R, del Zoppo GJ, Larrue V, Lesaffre E, Ringleb AP, Lorenzano S, Manelfe C, Bozzao L: Hemorrhagic transformation within 36 hours of a cerebral infarct: relationships with early clinical deterioration and 3-month outcome in the European Cooperative Acute Stroke Study I (ECASS I) cohort. Stroke 1999;30:2280-2284.

11 Hart RG, Easton JD: Hemorrhagic infarcts. Stroke 1986;17:586-589.

12 Okada Y, Yamaguchi T, Minematsu K, Miyashita T, Sawada T, Sadoshima S, Fujishima M, Omae T: Hemorrhagic transformation in cerebral embolism. Stroke 1989;20:598-603.

13 Fisher M, Adams RD: Observations on brain embolism with special reference to the mechanism of hemorrhagic infarction. J Neuropathol Exp Neurol 1951;10:92-94.

14 Sussman ES, Connolly ES Jr: Hemorrhagic transformation: a review of the rate of hemorrhage in the major clinical trials of acute ischemic stroke. Front Neurol 2013;4:69.

15 Berger C, Fiorelli M, Steiner T, Schäbitz WR, Bozzao L, Bluhmki E, Hacke W, von Kummer R: Hemorrhagic transformation of ischemic brain tissue: asymptomatic or symptomatic? Stroke 2001;32:1330-1335.

16 Toni D, Fiorelli M, Bastianello S, Sacchetti ML, Sette G, Argentino C, Montinaro E, Bozzao L: Hemorrhagic transformation of brain infarct: predictability in the first 5 hours from stroke onset and influence on clinical outcome. Neurology 1996;46:341-345.

17 Paciaroni M, Agnelli G, Corea F, Ageno W, Alberti A, Lanari A, Caso V, Micheli S, Bertolani L, Venti M, Palmerini F, Biagini S, Comi G, Previdi P, Silvestrelli G: Early hemorrhagic transformation of brain infarction: rate, predictive factors, and influence on clinical outcome: results of a prospective multicenter study. Stroke 2008; 39:2249-2256.

18 Bayramoğlu M, Karataș M, Leblebici B, Cetin N, Sözay S, Turhan N: Hemorrhagic transformation in stroke patients. Am J Phys Med Rehabil 2003;82:48-52. 